\title{
Exclusive Photoproduction of $\rho^{0}$ Meson with Leading Neutron at HERA
}

\section{Sergey Levonian ${ }^{* \dagger}$}

DESY, Notkestraße 85, 22607 Hamburg, Germany

E-mail: levonian@mail.desy.de

The first measurement of exclusive photoproduction of $\rho^{0}$ mesons associated with leading neutrons at HERA is presented. The data are taken with the H1 detector in the years 2006-2007 at $\sqrt{s}=319 \mathrm{GeV}$ and correspond to an integrated luminosity of $1.16 \mathrm{pb}^{-1}$.

$\rho^{0}$ mesons are reconstructed from their decays to pions in the central tracking chamber, while leading neutrons carrying a large fraction of the incoming proton momentum, $x_{L}>0.35$, are detected in the Forward Neutron Calorimeter. The phase space of the measurement is defined by the photon virtuality $Q^{2}<2 \mathrm{GeV}^{2}$, the total energy of the photon-proton system $20<W_{\gamma \mathrm{p}}<100$ $\mathrm{GeV}$ and the polar angle of the leading neutron, $\theta_{n}<0.75 \mathrm{mrad}$. The cross section of the reaction $\gamma p \rightarrow \rho^{0} n Y$, where $Y$ is a small mass system escaping undetected in the proton direction, is measured as a function of the neutron energy, of the effective mass of the $\pi^{+} \pi^{-}$system as well as of the transverse momentum squared, $p_{t}^{2}$, and of the pseudorapidity $\eta$ of $\rho$ meson.

The data are interpreted in terms of the double peripheral process, involving pion exchange at the proton vertex followed by elastic photoproduction of a $\rho$ meson on the virtual pion. In the framework of OPE approach elastic cross section of photon-pion scattering, $\sigma^{\mathrm{el}}\left(\gamma \pi^{+} \rightarrow \rho^{0} \pi^{+}\right)$ at average energy $\left\langle W_{\gamma \pi}\right\rangle=22 \mathrm{GeV}$ is extracted.

XXII. International Workshop on Deep-Inelastic Scattering and Related Subjects 28 April - 2 May 2014

Warsaw, Poland

\footnotetext{
*Speaker.

${ }^{\dagger}$ On behalf of the H1 Collaboration.
} 


\section{Introduction}

The aim of the analysis is to measure exclusive $\rho^{0}$ production on virtual pion in the photoproduction regime at HERA and to extract for the first time experimentally elastic $\gamma \pi$ cross section. In the Regge framework the events of such class are explained by the diagram shown in Fig. 1a which involves an exchange of two Regge trajectories in the process $2 \rightarrow 3$, known as Double Peripheral Process (DPP), or Double-Regge-pole exchange reaction [1]. Events of this type are modelled by the two-step Monte Carlo generator POMPYT [2] in which the virtual pion is produced at the proton vertex according to one of the available pion flux parametrisations. This pion then scatters elastically on the photon from the electron beam, thus producing vector meson ( $\rho^{0}$ in our case).

Diffractive dissociation of the proton into a system $Y$ (Fig. 1b) is an evident and major background to DPP. Using H1 detector capabilities in the forward region one can suppress such background to some level, but not completely. In particular, it contains an essentially irreducible part, originating from events where $Y=N^{*}$ decaying into $n+\pi^{+}$and leading to the identical final state as the process of interest. This background is modelled by the DIFFVM generator [3] and has to be subtracted from the data.

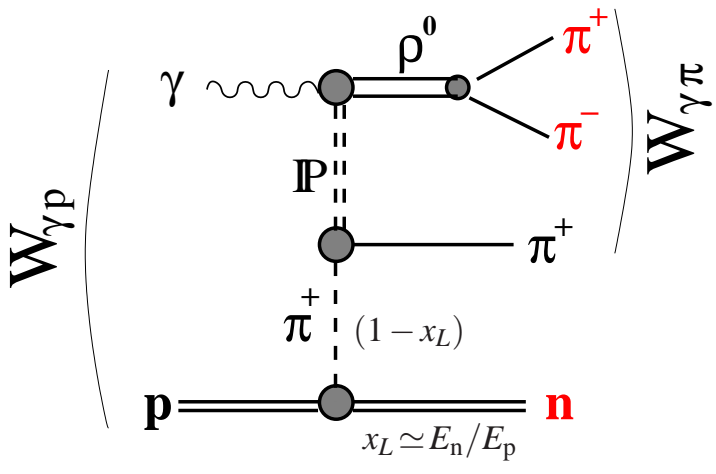

(a)

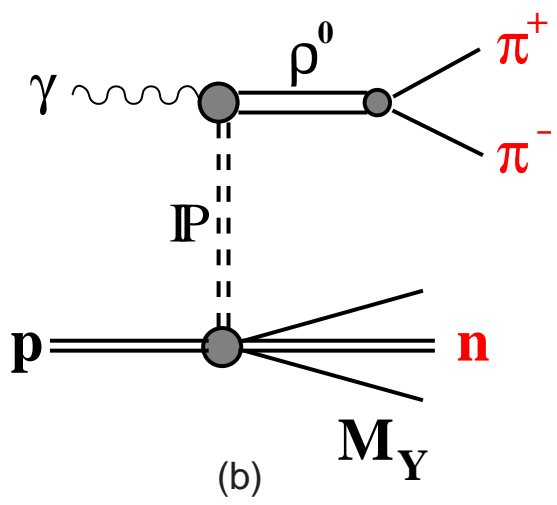

(b)

Figure 1: Generic diagrams for the two processes, contributing to exclusive photoproduction of $\rho$ mesons associated with leading neutrons at HERA. (a) Double peripheral process, involving $\pi$-exchange at the proton vertex, which is considered as a signal in present analysis. (b) Diffractive scattering in which neutron may be produced as a part of the proton dissociation system, $M_{Y}$, and which is treated as background to the events of class (a).

\section{Analysis Outline and Main Results}

The analysis is based on $\sim 6600$ events, containing only two charged pions from $\rho^{0}$ decay and a leading neutron with energy $E_{n}>120 \mathrm{GeV}$, and nothing else above noise level in the detector. The sample corresponds to an integrated luminosity of $1.16 \mathrm{pb}^{-1}$, collected by a special minimum bias track trigger in the years 2006-2007 at $\sqrt{s_{e p}}=319 \mathrm{GeV}$. The photon virtuality is limited to $Q^{2}<2$ $\mathrm{GeV}^{2}$ and according to $\mathrm{MC}$ its average value is $0.05 \mathrm{GeV}^{2}$. Hence we use WWA [4] formula to calculate photon flux from the electron and to convert $e p$ cross sections to $\gamma p$ ones.

After all selections the remaining background fraction in this sample is estimated as $0.36 \pm$ 0.06. Control plots illustrating the data description by the Monte Carlo models using this signal to 
background ratio are shown in Fig. 2. Since both POMPYT and DIFFVM cannot provide reliable absolute cross section prediction for such final state, only a shape comparison is possible.

\section{Exclusive photoproduction of $\rho^{0}$ with Forward Neutrons}
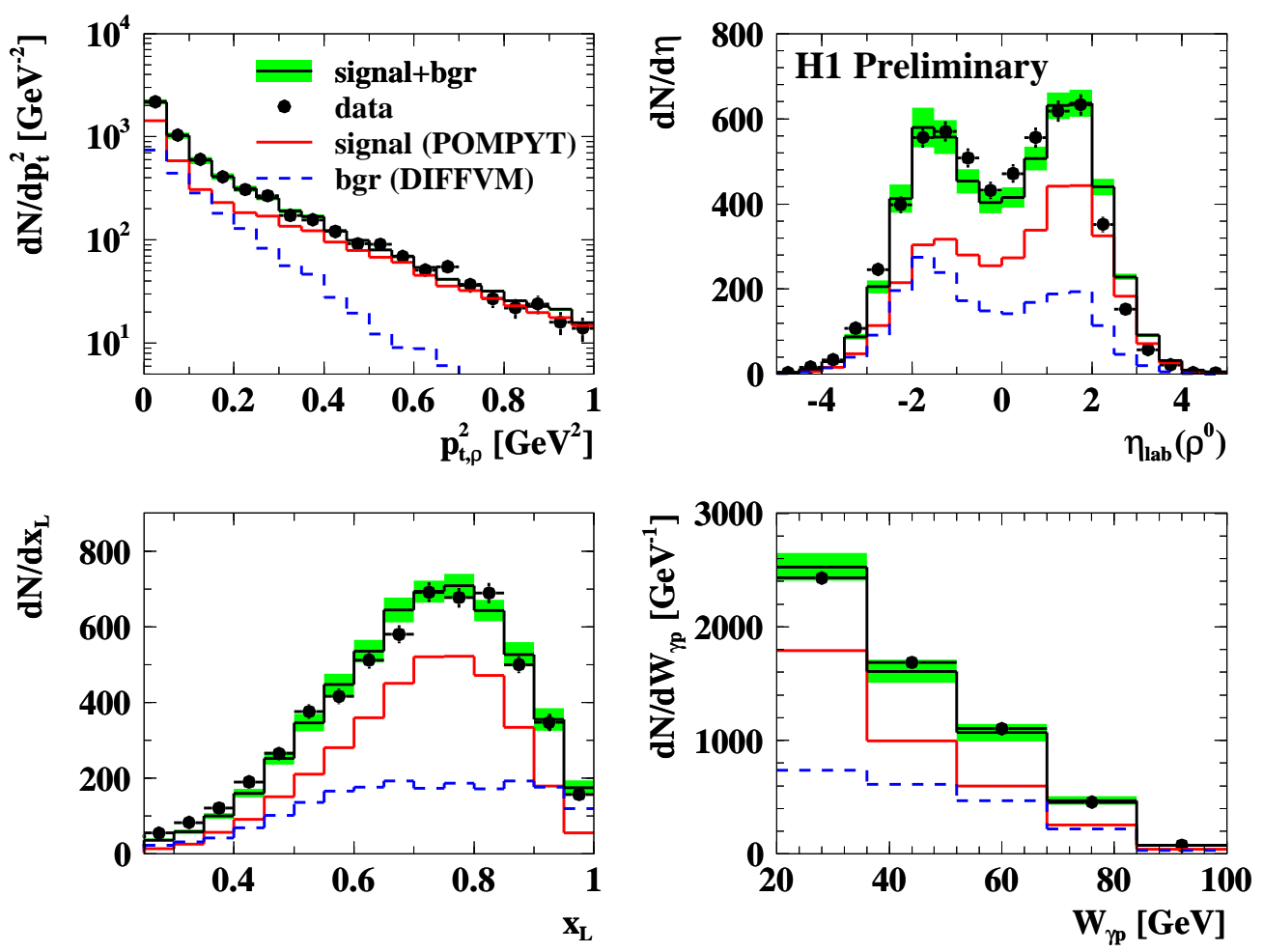

Figure 2: Distributions of $p_{t}^{2}$ and $\eta$ of $\rho^{0}, x_{L}$ of neutron and $W_{\gamma p}$ for data and Monte Carlo simulations normalised to the data. Data points are shown with statistical errors only. The shaded green band indicates the uncertainty in the estimated background fraction.

The effective mass distribution for two charged pions with $p_{t}>200 \mathrm{MeV}$ each and within the central detector range $20^{\circ}<\theta<160^{\circ}$ is shown in Fig. 3a. The distribution is corrected for the mass dependent detector efficiency. A fit is performed in the range $M_{\pi \pi}>0.4 \mathrm{GeV}$ using the Ross-Stodolsky parametrisation [5] for the $\rho^{0}$ meson shape and adding the contributions for the reflection from $\omega \rightarrow \pi^{+} \pi^{-} \pi^{0}$ and for the non-resonant background. The total contamination from the last two terms inside the analysis region $0.6<M_{\pi \pi}<1.1 \mathrm{GeV}$ was found at a negligible level, $(1.1 \pm 0.7) \%$. The fitted values of the mass, $M_{\rho}$, and the width, $\Gamma_{\rho}$, are in agreement with the nominal PDG values. The cross section is then calculated for the full range $2 m_{\pi}<M_{\pi \pi}<M_{\rho}+5 \Gamma_{\rho}$ using the resonant part only, represented by the relativistic Breit-Wigner function $B W_{\rho}\left(M_{\pi \pi}\right)$.

The Breit-Wigner shape is strongly distorted due to interference with non-resonant $\pi \pi$ production amplitude (dashed curve on Fig. 3). The strength of the distortion is $p_{t}$ dependent and can be characterised by the phenomenological skewing parameter, $n_{R S}$, as suggested by Ross and Stodolsky:

$$
\frac{d N\left(M_{\pi \pi}\right)}{d M_{\pi \pi}} \propto B W_{\rho}\left(M_{\pi \pi}\right)\left(\frac{M_{\rho}}{M_{\pi \pi}}\right)^{n_{R S}}
$$


Fig. $3 \mathrm{~b}$ shows $n_{R S}$ as a function of $p_{t}^{2}$ of $\rho^{0}$ for this measurement in comparison with previously published H1 [6] and ZEUS [7] results from elastic photoproduction of $\rho^{0}$.

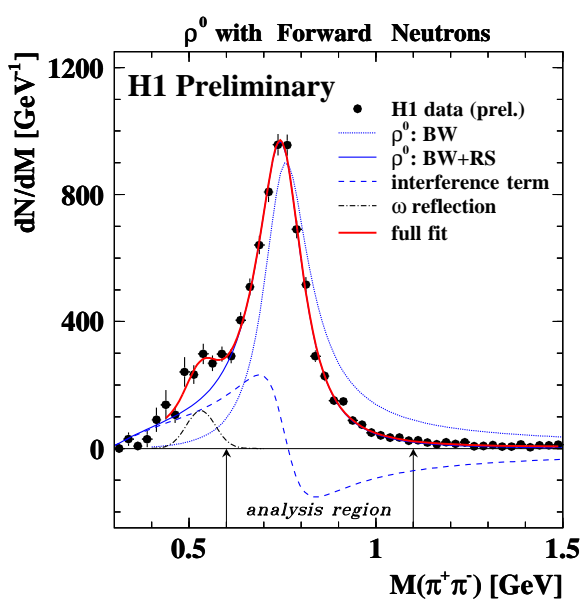

(a)

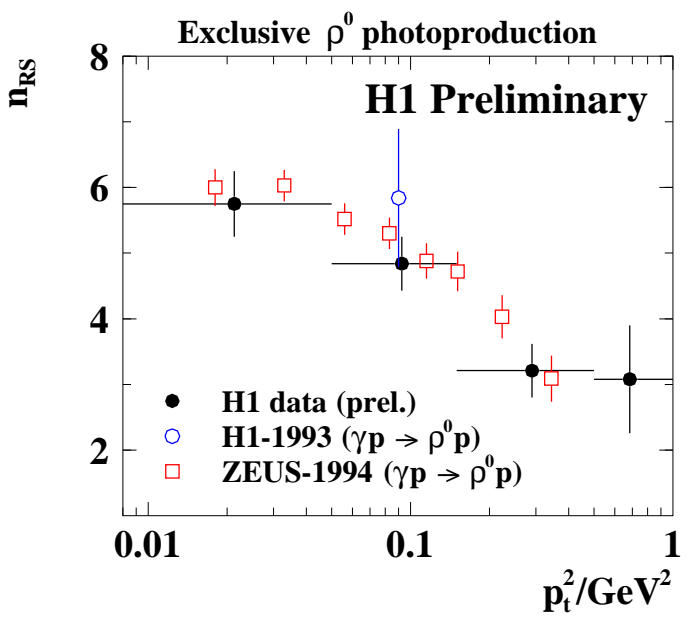

(b)

Figure 3: (a) - mass distribution of the $\pi^{+} \pi^{-}$system for elastic $\rho^{0}$ production with $p_{t}^{2}<1.0 \mathrm{GeV}^{2}$. The data points are corrected for the detector efficiency. The curves represent different components contributing to the measured distribution and the BW resonant part extracted from the fit to the data. Analysis region $0.6<M_{\pi^{+} \pi^{-}}<1.1 \mathrm{GeV}$ is indicated by vertical arrows. (b) - Ross-Stodolsky skewing parameter, $n_{R S}$, as a function of $p_{t}^{2}$ of $\pi^{+} \pi^{-}$system. Values measured in this analysis are compared to previously obtained results for elastic photoproduction of $\rho^{0}$ mesons, $\gamma p \rightarrow \rho^{0} p$, by H1 [6] and ZEUS[7] Collaborations.

The total cross section for the reaction $\gamma p \rightarrow \rho^{0} n\left(\pi^{+}\right)$is measured as

$$
\sigma_{\gamma p}=\left(280 \pm 6_{\text {stat }} \pm 46_{\text {sys }}\right) \mathrm{nb}
$$

for the phase space $20<W_{\gamma p}<100 \mathrm{GeV}, 0.35<x_{L}<0.95$ and $\theta_{n}<0.75 \mathrm{mrad}$.

In the one-pion-exchange (OPE) approximation the cross section for the leading neutron photoproduction can be expressed as a convolution of the pion flux and a photon-pion cross section:

$$
\frac{d \sigma_{\gamma p \rightarrow X n}\left(W^{2}, x_{L}, t\right)}{d x_{L} d t}=f_{\pi^{+} / p}\left(x_{L}, t\right) \cdot \sigma_{\gamma \pi^{+}}\left(\left(1-x_{L}\right) W^{2}\right)
$$

For small four momentum transfer squared, $t$, which is ensured in our case by the geometric acceptance of the Forward Neutron Calorimeter, the $p \rightarrow n$ transition amplitude is dominated by the lightest particle in the $t$-channel, the pion, and Eq.2.2 is a good approximation.

The differential cross section $\mathrm{d} \sigma_{\gamma p} / \mathrm{d} x_{L}$ is shown in Fig.4. Predictions from several $\pi$-flux models [8] are confronted with these data. Again, only a shape comparison is possible, since the $\gamma \pi$ cross section is not known. However, some models can be excluded even on the basis of this shape comparison. The remaining parametrisations of the pion flux which are compatible with the data are used to extract the $\gamma \pi$ cross section from this measurement. For the central value we use Holtmann flux [8] with coupling constant for charged pions, $g_{c} / 4 \pi=14.11 \pm 0.20$ [9]. Integrated flux values, $\Gamma_{\pi^{+}}=\int f_{\pi^{+} / p}\left(x_{L}, t\right) \mathrm{d} x_{L} \mathrm{~d} t$, for the region of measurement, $0.35<x_{L}<$ $0.95, p_{t, n}<x_{L} \cdot 0.69 \mathrm{GeV}$, still differ significantly between different parametrisations, leading to a model uncertainty of $\sim 25 \%: \Gamma_{\pi^{+}}=0.138_{-0.027}^{+0.039}$. 

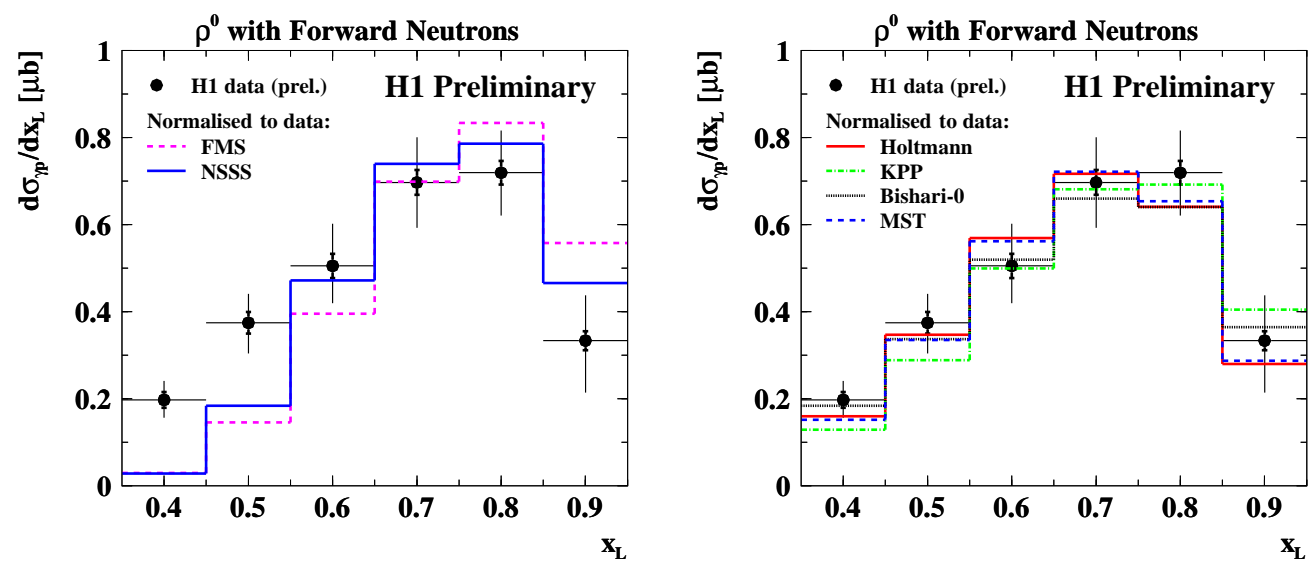

Figure 4: Differential cross section $\mathrm{d} \sigma_{\gamma p} / \mathrm{d} x_{L}$ compared to the predictions based on different versions of pion fluxes [8]. The data points are shown with statistical (inner error bars) and total (outer error bars) uncertainties, excluding overall normalisation error of 5.9\%. All predictions are normalised to the data.

The extracted cross section for the mean value of $\left\langle W_{\gamma \pi}\right\rangle=22 \mathrm{GeV}$ is found to be

$$
\sigma\left(\gamma \pi^{+} \rightarrow \rho^{0} \pi^{+}\right)=\left(2.03 \pm 0.34_{\exp } \pm 0.51_{\text {model }}\right) \mu \mathrm{b}
$$

where the first error represents the total experimental uncertainty and the second one is due to the pion flux uncertainty. Taking Regge-fitted value of $\sigma\left(\gamma p \rightarrow \rho^{0} p\right)=9.5 \pm 0.5 \mu \mathrm{b}$ at corresponding energy, which is an interpolation between fixed target and HERA measurements (see e.g. Fig. 10 in [7]) one can obtain for the ratio of elastic $\rho^{0}$ photoproduction on pion and on proton

$$
r=\frac{\sigma_{\gamma \pi}^{\mathrm{el}}}{\sigma_{\gamma p}^{\mathrm{el}}}=0.21 \pm 0.06
$$

This can be compared to the similar ratio, but for total cross sections, estimated by ZEUS [10]: $r=\sigma_{\gamma \pi}^{\text {tot }} / \sigma_{\gamma p}^{\text {tot }}=0.32 \pm 0.03$. Both values are lower than naïvely expected $r=2 / 3$ from AQM considerations.

Fig. 5 shows the energy dependence of exclusive $\rho^{0}$ production with a leading neutron, $\sigma_{\gamma p \rightarrow \rho^{0} n \pi^{+}}\left(W_{\gamma p}\right)$. Regge motivated fit $\sigma_{\gamma p} \propto W^{\delta}$ yields a value of $\delta=-0.33 \pm 0.06_{\text {stat }} \pm 0.13_{\text {sys }}$. POMPYT MC predicts different trend, typical for Pomeron exchange only.

Finally, in Fig. 6 the differential distribution $\mathrm{d} \sigma / \mathrm{d} p_{t}^{2}$ for $\rho$ mesons is shown. It exhibits two distinctly different exponential slopes, $b_{1}=25.0 \pm 6.1 \mathrm{GeV}^{-2}$ and $b_{2}=3.28 \pm 0.31 \mathrm{GeV}^{-2}-\mathrm{a}$ feature known to be a characteristic for DPP reactions [1].

\section{Summary}

Photoproduction cross section for exclusive $\rho^{0}$ production associated with leading neutron is measured for the first time at HERA. The elastic photon-pion cross section, $\sigma\left(\gamma \pi^{+} \rightarrow \rho^{0} \pi^{+}\right)$, at $\langle W\rangle=22 \mathrm{GeV}$ has been extracted in the OPE approximation. The differential cross section $\mathrm{d} \sigma / \mathrm{d} p_{t, \rho}^{2}$ shows the behaviour typical for exclusive double peripheral processes. 


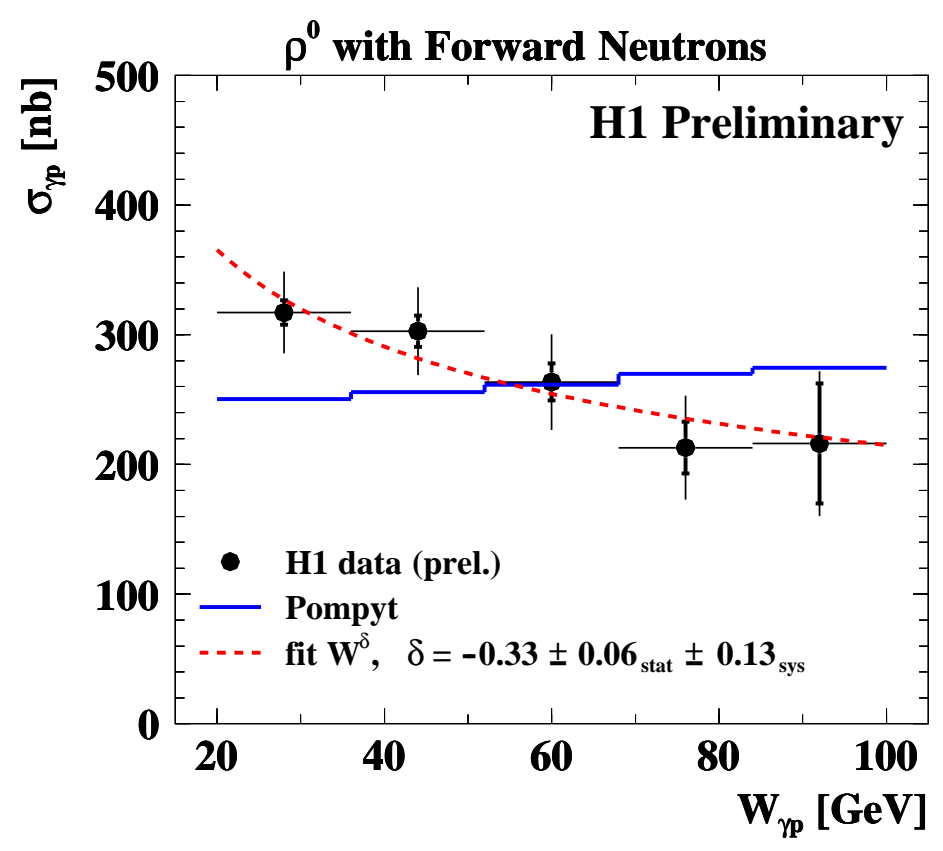

Figure 5: Cross section of the reaction $\gamma p \rightarrow \rho^{0} n \pi^{+}$as a function of $W_{\gamma p}$ compared to prediction from POMPYT MC, which is normalised to the data. The dashed curve represents the Regge motivated fit $\sigma \sim W^{\delta}$ with $\delta=-0.33 \pm 0.06_{\text {stat }} \pm 0.13_{\text {sys. }}$. The data points are shown with statistical (inner error bars) and total uncertainties (outer error bars) excluding overall normalisation error of $5.9 \%$

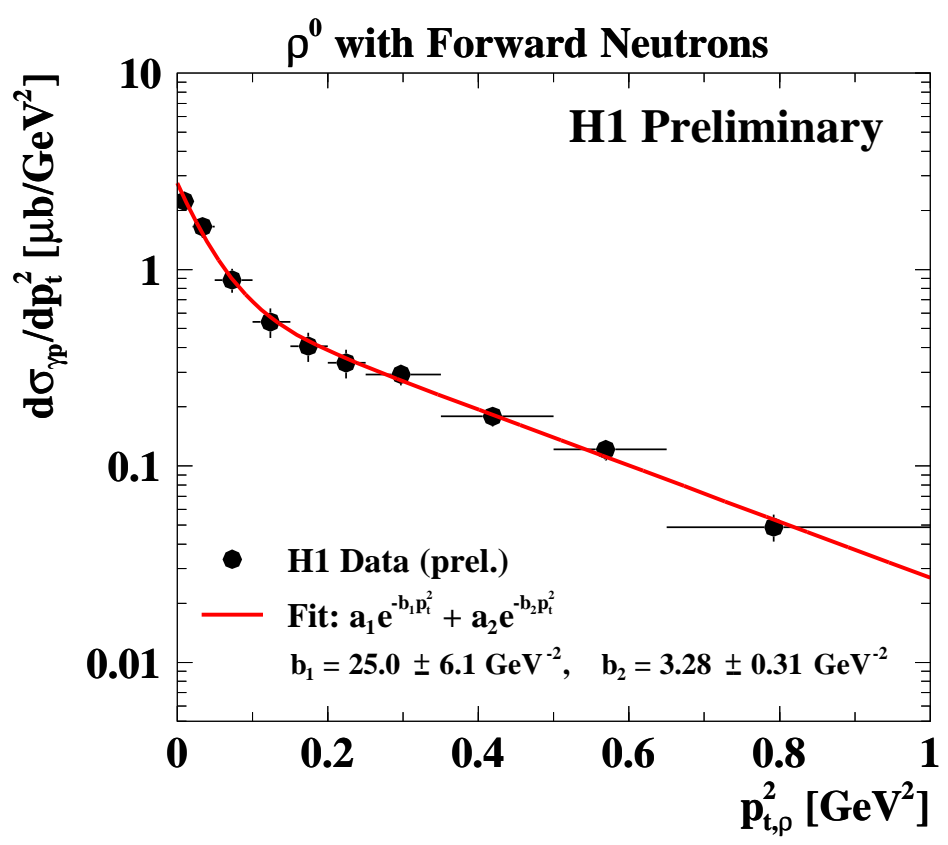

Figure 6: Differential cross section $\mathrm{d} \sigma_{\gamma p} / \mathrm{d} p_{t}^{2}$ of $\rho^{0}$ mesons fitted with the sum of two exponential functions. The values of slopes are characteristic for double peripheral processes [1] in which an exchange of two Regge trajectories is involved. 


\section{References}

[1] N.F. Bali, G.F. Chew and A. Pignotti, Multiple-Production Theory Via Toller Variables, Phys. Rev. Lett. 19 (1967) 614;

G.F. Chew and A. Pignotti, Multiperipheral Bootstrap Model, Phys. Rev. 176 (1968) 2112;

E. L. Berger, Reggeized Double-Peripheral-Model Analysis of Three-Body Final-State Processes, Phys. Rev. 179 (1969) 1567;

N.P. Zotov and V.A. Tsarev, Diffractive Dissociation and Drell-Hiida-Deck Model, (In Russian) Fiz.Elem.Chast.Atom. Yadra 9 (1978) 650.

[2] P. Bruni and G. Ingelman, Diffractive hard scattering at e $p$ and $p$ anti-p colliders, in proceedings of the Europhysics Conference, C93-07-22, Marseille, France (1993) 595.

[3] B. List and A. Mastroberardino, DIFFVM - A Monte Carlo generator for diffractive processes in ep scattering, Proc. of the Workshop on Monte Carlo Generators for HERA Physics, eds. A.T. Doyle et al., DESY-PROC-1999-02 (1999) 396.

[4] E.J. Williams, Nature of the high-energy particles of penetrating radiation and status of ionization and radiation formulae, Phys. Rev. 45 (1934) 729;

C.F. Weizsäcker, Radiation emitted in collisions of very fast electrons, Z. Phys. 88 (1934) 612;

S. Frixione et al., Improving the Weizsacker-Williams approximation in electron - proton collisions, Phys. Lett. B319 (1993) 339.

[5] M. Ross and L. Stodolsky, Photon dissociation model for vector meson photoproduction, Phys. Rev. 149 (1966) 1172.

[6] S. Aid et al. [H1 Collaboration], Elastic Photoproduction of $\rho^{0}$ Mesons at HERA, Nucl. Phys. B463 (1996) 3 [hep-ex/9601004].

[7] J. Breitweg et al. [ZEUS Collaboration], Elastic and Proton-Dissociative $\rho^{0}$ Photoproduction at HERA, Eur. Phys. J. C2 (1998) 247.

[8] (a) M. Bishari, Pion exchange and inclusive spectra, Phys. Lett. B38 (1972) 510;

(b) H. Holtmann, A. Szczurek and J. Speth Flavor and spin of the proton and the meson cloud, Nucl. Phys. A596 (1996) 631; M.Przybycien, A.Szczurek and G.Ingelman, Properties of HERA events from DIS on pions in the proton, Z. Phys. C74 (1997) 509;

(c) B.Kopeliovich, B.Povh and I.Potashnikova, Deep inelastic electroproduction of neutrons in the proton fragmentation region, Z. Phys. C73 (1996) 125;

(d) W. Melnitchouk, J. Speth and A.W.Thomas, Dynamics of light anti-quarks in the proton, Phys. Rev. D59 (1999) 014033;

(e) L. Frankfurt, L. Mankiewicz and M. Strikman, Low Limit on the Slope of $N N \pi$ and $N \Delta \pi$ Vertex Form-factors From Deep Inelastic Lepton Scattering, Z. Phys. A334 (1989) 343;

(f) N.N. Nikolaev, W.Schäfer, A. Szczurek and J. Speth, Do the E866 Drell-Yan data change our picture of the chiral structure of the nucleon? Phys. Rev. D60 (1999) 014004.

[9] T.E.O. Ericson, B. Loiseau and A.W. Thomas, Determination of the pion-nucleon coupling constant and scattering lengths, Phys. Rev. C66 (2002) 014005.

[10] S. Chekanov et al. [ZEUS Collaboration], Leading Neutron Production in ep collisions at HERA, Nucl. Phys. B637 (2002) 3. 